\title{
Multiplexing Control of a Multichannel Piezoelectric
}

\section{Deformable Mirror}

\author{
Hong Song1, 2, Aleksey N. Simonov2 and Gleb Vdovin2 \\ 1.Fudan University, 200433, Shanghai, P.R.China, \\ 2.Electronic Instrumentation, Delft University of Technology, Mekelweg 4, 2628 CD, Delft, \\ The Netherlands
}

\begin{abstract}
Addressing of massive arrays of piezoelectric actuators is usually achieved by using separate high-voltage output drivers, one per channel. This approach applied to high-order adaptive optics systems results in complex, expensive and vulnerable to handling abuse driver electronics, hardly scalable to $10^{3}-10^{4}$ actuators. To reduce the number of identical electronic units and simplify the control, we propose sequential multiplexing of piezoelectric actuators. The relatively large capacitance inherent in mirror piezo-actuators allows for storage of charge (high voltage) on a disconnected actuator retaining its displacement, while other actuators are addressed. As a demonstrator a 12-channel piezoelectric deformable mirror driven by a single high-voltage amplifier has been characterized experimentally. The multiplexing of actuators was accomplished by miniature optical switches. Temporal stability of $\sim \lambda / 100$ was demonstrated at multiplexing frequency of $700 \mathrm{~Hz}$ with a full-range $\sim 2 \mu \mathrm{m}$ inter-actuator stroke. The developed approach can be scaled to higher-order deformable mirrors.
\end{abstract}

Keywords: Adaptive optics, deformable mirror, multiplexing control.

\section{INTRODUCTION}

A deformable mirror (DM) is known to be one of the key parts of adaptive optics (AO) systems. ${ }^{1,2}$ A number of scientific ${ }^{3,4}$, medical ${ }^{5,6}$, and industrial ${ }^{2,5,7}$ applications of adaptive optics (AO) being developed at present require relatively inexpensive, reliable, high-quality, e.g. with many degrees of freedom and simple in operation wave-front correctors ${ }^{8}$. In some cases DMs may include hundreds or even thousands of actuation channels, that are becoming quite common nowadays especially in astronomical AO systems ${ }^{1,2,4,9-12}$. Typically, each DM channel is driven by an individual electronic control unit. In the case of piezoelectric actuators, high-voltage amplifiers with the output voltage ranging from $\sim 10^{2} \mathrm{~V}$ to $10^{3} \mathrm{~V}$ and bandwidth of $\sim 0-10^{3} \mathrm{~Hz}$ are required.

Figure 1 below shows the schematic of a typical piezoelectric DM, which consists of the mirror surface, piezoelectric actuators, substrate (mirror base) and driver electronics. Actuators elongate or shrink when control voltages are applied to the electrodes. This results in the desired deformation of the mirror surface.

5th International Workshop on Adaptive Optics for Industry and Medicine, edited by Wenhan Jiang, Proc. of SPIE Vol. 6018, 60181F, (2005) · 0277-786X/05/\$15 · doi: 10.1117/12.669385 
Individual low-level control signals $\left\{V_{i 1} \ldots V_{i N}\right\}$ can be generated by a computer-integrated D/A board in the course of the processing of data acquired from a wave-front sensor or data produced in computations. In turn, to get the desirable deformation (of the order of several $\mu \mathrm{m}$ for visible range AO systems) of the mirror surface, high voltages (several hundred volts) should be applied to actuators. Amplification of the control signals is usually accomplished by individual highvoltage amplifiers (HVA).

In the case of high-order $\mathrm{AO}$ systems, the number of actuation channels may exceed $10^{3}-10^{4}$, resulting in significant amount of electronics and complex multi-wire connection for a high-order DM. All this makes the whole AO system rather bulky, vulnerable to handling abuse and very expensive. Serious problems occur with weight, volume and power dissipation so that the high-order deformable mirror becomes extremely complex in operation. The total cost of the driver electronics for a high-order adaptive optics system may account for two thirds of the total system cost. ${ }^{2}$

An attempt to simplify the DM electronics by sequential addressing of actuators was made by Kibblewhite et al. ${ }^{13}$ They build a 59-channel piezoelectric faceplate mirror driven by several high-voltage amplifiers (HVA) via 16 high-voltage switches assembled from discrete components. The overall refresh rate was $4 \mathrm{kHz}$ with the maximum voltage swing of $\sim 40 \mathrm{~V}$ on the selected actuator per update. Due to the implementation and control complexity as well as imperfection of the multiplexer in terms of power saving, this approach has not won acceptance. ${ }^{2}$

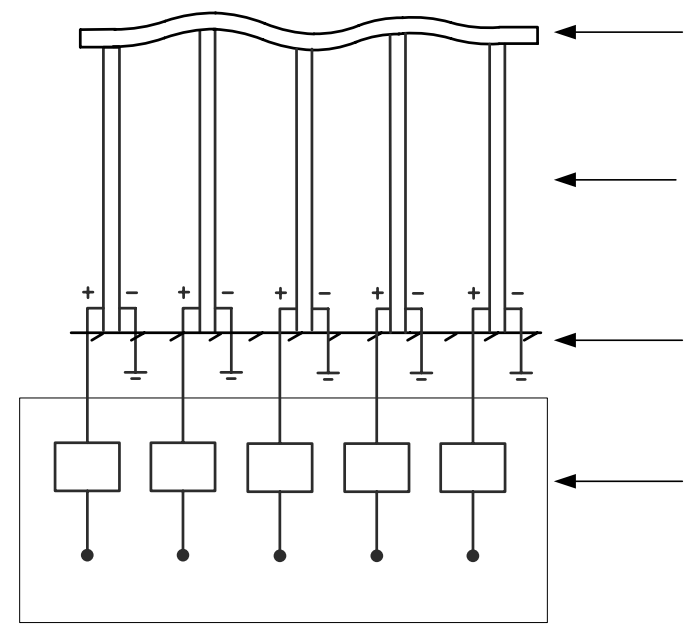

Fig.1. Typical piezoelectric DM with individual channels control.

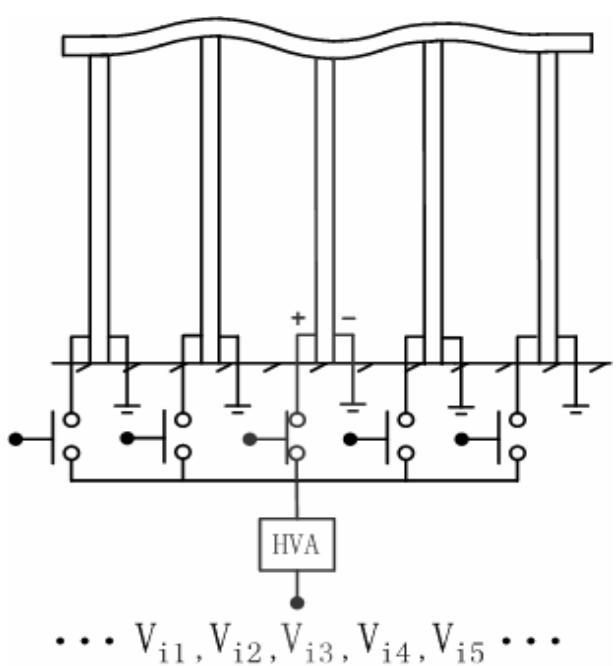

Fig.2. Principle of multiplexing control. 
In this work we report on the possibility of high-order piezoelectric DM with internal multiplexing and inexpensive driving electronics that utilizes an algorithm of adaptive addressing. The principle of this approach is that the addressing of piezoelectric actuators is accomplished not continuously but as needed through the look-up table to follow the required DM figure. The simplicity and compactness of the elaborated driver electronics allows it to be integrated with the deformable mirror.

\section{MULTIPLEXING CONTROL}

\subsection{PRINCIPLE OF MULTIPLEXING CONTROL}

In the multiplexing mode, only one HVA can be used for an $N$-channel DM and $N$ switches should be employed (see Fig. 2 above) to allow connecting the output of the HVA with DM channels. At one moment only one channel is connected to the HVA by turning on corresponding switch, while others are disconnected. In the next moment, another switch is turned on and the rest are disconnected. By doing this repeatedly and synchronizing the switches and the HVA outputs, $N$ channels of the mirror can be driven by a single HVA.

It should be noted that a multiplexing technique with subsequent addressing of DM channels requires a pre-generated lookup table (a data array) in the control algorithm that contains values of DM signals for the next addressing cycle. This feature can be used to optimize the overall performance of the mirror by means of adaptive addressing. Taking into account large leakage time $\left(t_{l} \sim 10 \mathrm{~s}\right.$ for the actuator capacitance of $\left.\sim 10 \mathrm{nF}\right)$ of the piezoelectric actuators, the DM multiplexing can be organized in a way that only required actuators are addressed in every cycle, while others are updated at the slowest possible rate $\left(\sim 1 / t_{l}\right)$ to retain their size.

\subsection{IMPLEMENTATION OF THE CONTROL SYSTEM}

Figure 3 presents the block diagram of the 12-channel multiplexed DM with driving electronics and its implementation. The whole system consists of the following parts: (1) low-voltage control D/A board, (2) wide-band HVA, (3) array of opticallycontrolled switches, (4) 12-channel DM.

A computer-integrated D/A board from OKO Technologies ${ }^{14}$ is used as the low-voltage signal generator, which is capable of converting 8-bit binary values from the control program into analog output for 24 separate channels. The output voltage can be trimmed continuously in the range $0-10 \mathrm{~V}$ for the all channels with the use of standard programming software $(\mathrm{C}++$, Labview etc. ). The DC voltage gain of the unipolar FET-based HVA is 60 , its bandwidth (-3dB) measured under a singe piezo-actuator load $\left(C_{a}=12 \mathrm{nF}\right.$ ) is about $20 \mathrm{kHz}$ and the output range is $0-300 \mathrm{~V}$. The HVA utilizes standard inexpensive electronic components and is assembled on a 30x60 $\mathrm{mm}$ PCB that can be incorporated in the mirror housing. 
The multiplexer includes 12 miniature (DIP6 packaging) high-voltage triacs with optically-isolated (LED-based) low-level controls. They can be switched on/off by applying high or low-level voltages (in our case, 5V and 0V, respectively). Due to the weak current driving capability of the D/A board, each optotriac is driven through a single-transistor current buffer. The multiplexer board integrates current buffers with the optotriacs.

A 12-channel piezoelectric DM with a clear aperture of $25.4 \mathrm{~mm}$ was supplied by OKO Technologies. ${ }^{14}$ The mirror uses standard $3.2 \mathrm{~mm}$ diameter and $30 \mathrm{~mm}$ long tubular lead-zirconate-titanate (PZT) actuators positioned in a rectangular grid with $7 \mathrm{~mm}$ pitch. The measured in situ capacitance $C_{a}$ of PI actuators is $\sim 12 \mathrm{nF}$ and their $1 / e$ leakage time $t_{l}$ exceeds $30 \mathrm{~s}$. The DM mirror has a full stroke of $\sim 7 \mu \mathrm{m}$ (hysteresis less than $9 \%$ ) and an inter-actuator stroke of $\sim 2 \mu \mathrm{m}$, all measured in the $0-300 \mathrm{~V}$ range.

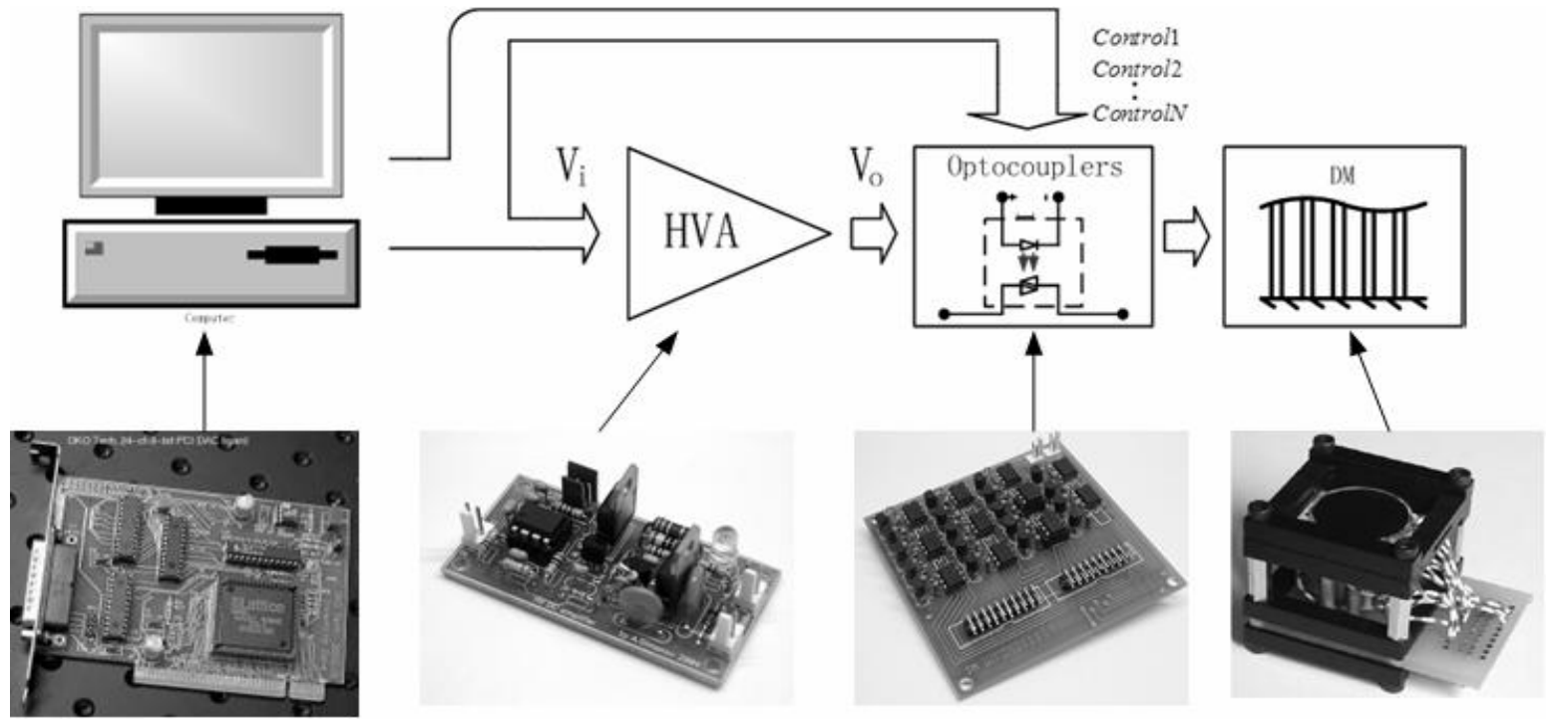

Fig.3. Block diagram of the control system and its implementation.

Because of the limited bandwidth of the HVA, turn on/off delays of the optotriacs and charge/discharge delays of the piezoactuators, the DM multiplexing and HVA output need to be accurately timed in order to achieve optimal performance of the DM. Otherwise, if there is no synchronization between the controls, inter-channel cross-talks occur resulting in the DM surface inaccuracy.

\section{EXPERIMENT}

To investigate how the mirror surface stability depends on the multiplexing regimes, we assembled the optical setup shown in figures 4 and 5 . The light beam of a He-Ne laser operating at $\lambda \cong 633 \mathrm{~nm}$ is attenuated by a polarizer (P) and then after passing through the spatial filter (SF) with a $50-\mu \mathrm{m}$ pinhole is split by a $50 / 50$ beam splitter $\left(\mathrm{SP}_{1}\right)$. Part of the collimated 
beam illuminates a low-finesse asymmetric Fabry-Perot interferometer formed by the DM mirror and a 50/50 beam splitter $\left(\mathrm{BS}_{2}\right)$. These elements are placed on a vibration isolated mount that permits accurate measurement of the DM displacement $d$. The light reflected from the interferometer is picked up by $\mathrm{BS}_{1}$ and through the imaging optics (IO) goes to a CCD or photodiode if the relatively fast movements of the mirror surface have to be determined. The CCD allows taking the interferograms of the DM influence functions and qualitative estimation of the mirror figure stability (see inset pictures in Fig. 4).
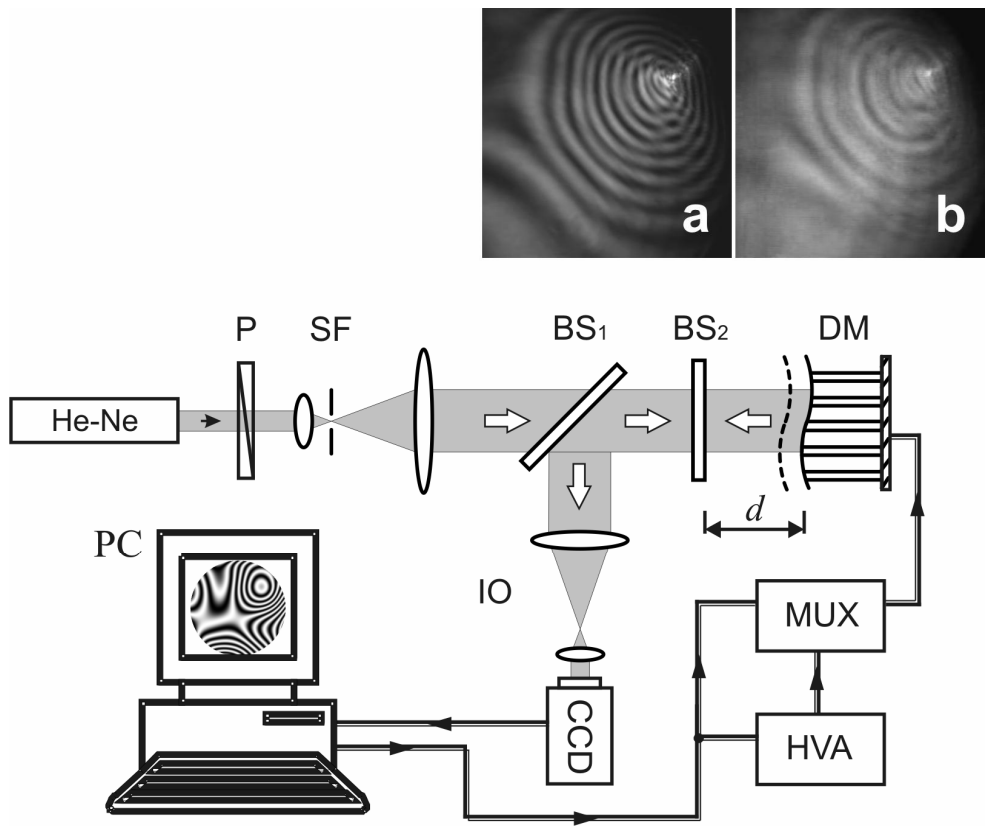

Fig. 4. Experimental setup. P, polarizer; SF, spatial filter; $\mathrm{BS}_{1}, \mathrm{BS}_{2}$, beam splitters; IO, imaging optics; DM, deformable mirror; HVA, high-voltage amplifier; MUX, channel multiplexer. Pictures above represent averaged over $1 \mathrm{~s}$ period interferograms of the $\mathrm{DM}$ at (a) low refresh frequency $F=5 \mathrm{~Hz}$ and (b) high refresh frequency $F=1700 \mathrm{~Hz}$, above the threshold value $F_{t h} \sim 1500 \mathrm{~Hz}$.

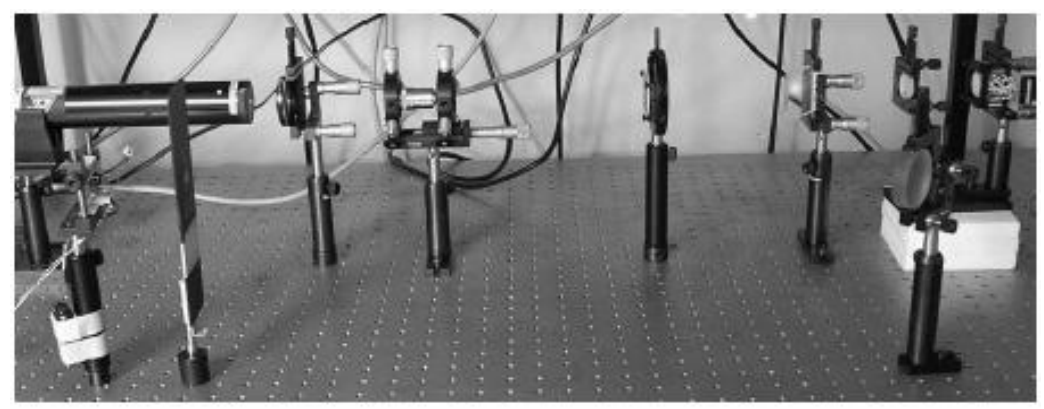


Fig. 5. Optical setup implementation.

The sequential multiplexing of DM clearly indicates that due to the limited bandwidth of the driver electronics (HVA settling time, optotriac on/off-times) and mechanical resonance of the mirror, there is a threshold frequency $\left(F_{t h}\right)$ at which the multiplexing becomes ineffective. Inset pictures in Fig.4 present two extreme cases: (a) the multiplexing frequency $(F)$ is below $F_{t h}$ and (b) $F$ exceeds $F_{t h}$. In this experiment a voltage of $250 \mathrm{~V}$ was applied to a single actuator, whereas others actuators were kept at $0 \mathrm{~V}$. The threshold frequency of multiplexing was found to be $F_{t h} \sim 1500 \mathrm{~Hz}$.

Change of the voltage applied to the piezoelectric actuators leads to displacement of the mirror surface $(\Delta d)$ and shift of the interference pattern. The resulting variation of light intensity can be used for quantitative estimation of the mirror figure stability.

To evaluate the DM surface displacement $\Delta d$ from its static position $\left(d_{0}\right)$ as a function of multiplexing frequency $F$ we employed the analytical expression for the total reflection coefficient $(R)$ of asymmetric Fabry-Perot interferometer versus its length $(d)$, see for example ${ }^{15}$, i. e. the distance between $\mathrm{BS}_{2}$ and $\mathrm{DM}$ :

$$
R=\left|\frac{-\sqrt{R_{1}}+\sqrt{R_{2}} \exp (2 i k d)}{1-\sqrt{R_{1} R_{2}} \exp (2 i k d)}\right|^{2}
$$

where $k=2 \pi / \lambda$ is the wave number; $R_{1}, R_{2}$ are the reflectivities of $\mathrm{BS}_{2}$ and $\mathrm{DM}$, respectively. The voltage required for a single actuator-induced $\lambda / 2$ displacement is measured to be $U_{\lambda / 2} \cong 35 \mathrm{~V}$. Thus, the required dependence of $\Delta d$ on $F$ can be obtained from Eq. (1) using $U_{\lambda / 2}$ value, provided the voltage applied to a specific actuator $\left(U_{a}\right)$ does not exceed $U_{\lambda / 2}$. Taking $d=d_{0}+\Delta d,|\Delta d|<<d_{0}$, where $d_{0}$ is the operation point of the interferometer, and assuming that $\Delta d$ follows $U_{a}$ linearly, e. i. $\Delta d \sim U_{a}$, we can obtain the following expression for the intensity of reflected light:

$$
I=I_{0}\left\{1+b\left(d_{0}, R_{1}, R_{2}\right) \times U_{a}\right\}
$$


where $b \sim d I / d U_{a}$ is the interferometer sensitivity slope (function of $d_{0}, R_{1}, R_{2}$ ) and $I_{0}$ is the reflected intensity at the interferometer output at $d=d_{0}$. Figure 6 below represents the reflected intensity versus relative displacement $d / \lambda$ for the case when $U_{a} \sim U_{a}^{(0)} \sin (2 \pi F t)$, and hence: $\Delta d \sim \sin (2 \pi F t)$.

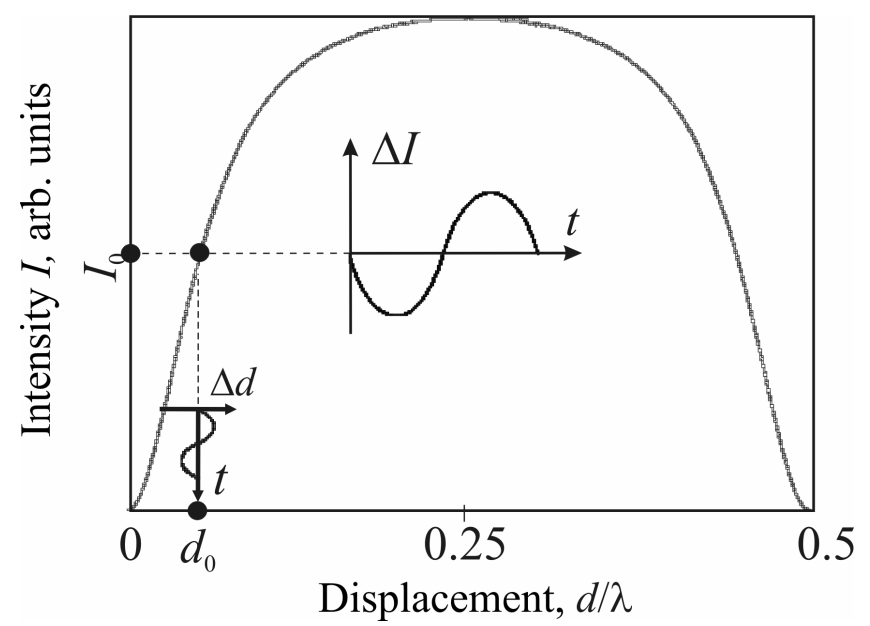

Fig. 6. Reflected light intensity from the interferometer as a function of its relative length $d / \lambda$.

\section{RESULTS}

Figure 7 below shows the measured single-actuator induced displacement of the mirror surface $\Delta d$ as a function of multiplexing frequency $F$. In this experiment, a voltage of $20 \mathrm{~V}$ is applied to a single piezoactuator, whereas other actuators are kept at $0 \mathrm{~V}$. As clearly seen, an rms figure stability of $\sim \lambda / 100$ is attained for the multiplexing frequencies in the range up to $3.5 \mathrm{kHz}$. 


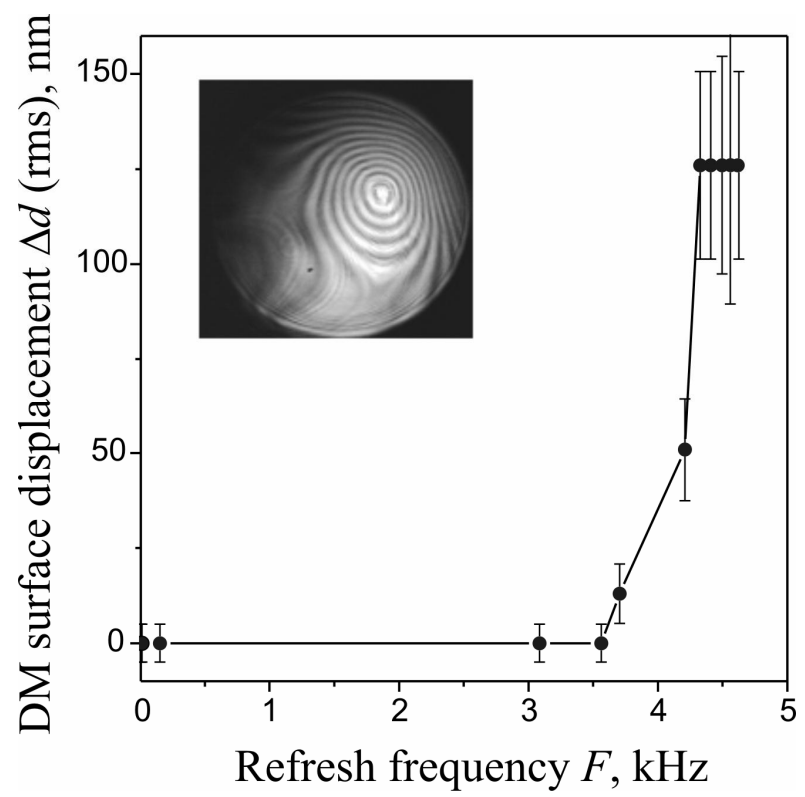

Fig.7. Multiplexing error: deviation of the DM from its static shape versus frequency. Inset shows the interferogram of the DM influence function.

The performance of the mirror has been examined in the most extreme conditions of multiplexing when there is a large voltage difference $\left({ }^{i a}\right)$ between the adjacent DM actuators. Figure 8 shows the threshold frequency of multiplexing $F_{t h}$ as a function of $U_{i a}$. The maximum inter-actuator stroke across the whole DM aperture is achieved with the following set voltages $\left\{U_{i a}, 0, U_{i a}, 0, U_{i a}, 0, U_{i a}, 0, U_{i a}, 0, U_{i a}, 0\right\}$ applied to PZT actuators of the 12-channel DM. As seen, the maximum mechanical inter-actuator stroke of $\sim 2 \mu \mathrm{m}\left(U_{i a}=300 \mathrm{~V}\right)$ is attained at $F<F_{t h}=700 \mathrm{~Hz}$, whereas the interstroke of $\sim 0.1 \mu \mathrm{m}\left(U_{i a}=12 \mathrm{~V}\right)$ limits $F_{\text {th }}$ to $\sim 3.6 \mathrm{kHz}$. The corresponding Nyquist frequencies are $350 \mathrm{~Hz}$ and $1.8 \mathrm{kHz}$, respectively. These values meet or surpass of typical atmospheric changes that are of the order of $0.1 \mu \mathrm{m}$ for $10 \mathrm{~ms} 2,13$. The DM threshold frequency curve in Fig.8 indicates that depending on the required deformation, the update rate can be adjusted. Thus, with the program look-up table the overall DM performance can be optimized by appropriate choice of update rate for every DM channel. 


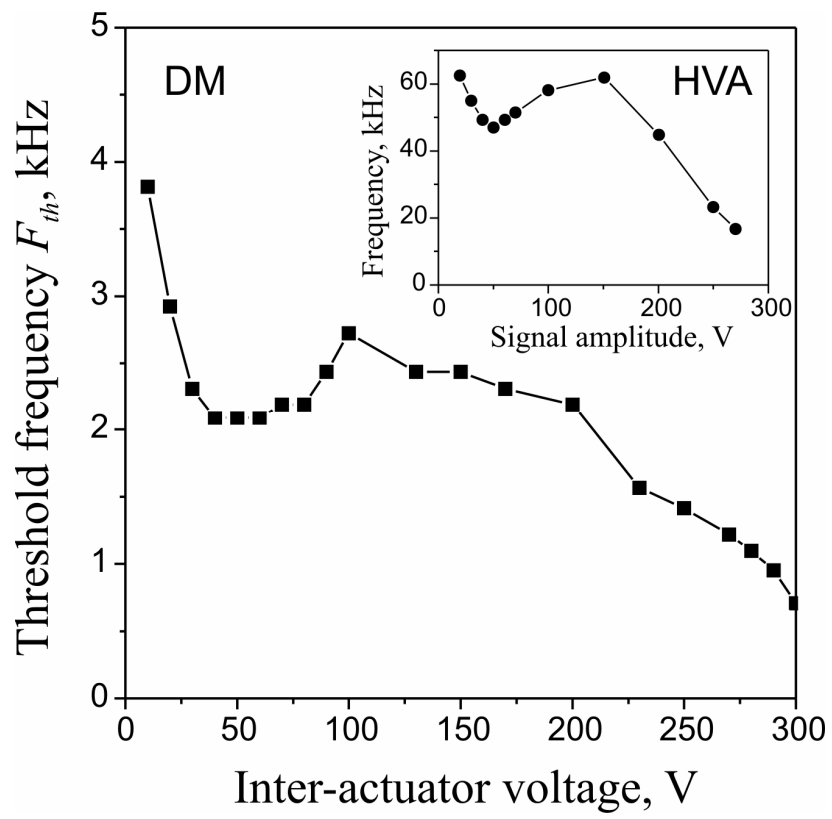

Fig. 8. Threshold multiplexing frequency for the 12-channel DM versus inter-actuator voltage swing. Inset represents the output frequency-voltage response of the HVA loaded by a singe PI piezoactuator.

\section{CONCLUSION}

We have presented multiplexing control for the piezoelectric deformable mirrors. To retain the required DM figure the adaptive address of piezoelectric actuators through the program-based look-up table can be implemented. This permits optimizing the mirror performance and reducing the total power consumption as only those actuators are addressed in the subsequent cycle that are needed. The multiplexing control has been demonstrated using a demonstrator 12-channel facesheet piezoelectric mirror. An rms figure stability of $\sim \lambda / 100$ is obtained for the multiplexing frequencies $<3.5 \mathrm{kHz}$. The frequencydependent mechanical inter-actuator stroke of the mirror reaches $\sim 2 \mu \mathrm{m}$ and decays to $\sim 0.1 \mu \mathrm{m}$ as the multiplexing frequency increases from $700 \mathrm{~Hz}$ to $3.6 \mathrm{kHz}$. The simplicity and small size of the driving electronics employing optotriac high-voltage switches allows it to be integrated with the DM.

The adaptive multiplexing (utilizing miniature optotriac switches) can be scaled to piezoelectric DMs with as much as $10^{2} \sim 10^{3}$ control channels resulting in mirror-integrated inexpensive driving electronics. Compared with a traditional control method, this approach has great advantages in respect of power dissipation, volume cost and scalability and can be suitable for the majority of applications in high-order AO systems.

\section{REFERENCES}


1. R.K. Tyson, Principles of adaptive optics (Boston: Academic Press, 1998), Chap. 6.

2. R.K. Tyson (ed.), Adaptive optics engineering handbook (New York, N.Y.: Marcel Dekker, Inc., 2000), Chap. 2, 5, 7 and 8 .

3. R. Bartels, S. Backus, E. Zeek, L. Misoguti, G. Vdovin, I. P. Christov, M. M. Murnane, and H. C. Kapteyn, “Shapedpulse optimization of coherent emission of high-harmonic soft X-rays”, Nature , 406, 164 - 166, 2000.

4. P.A. Knutsson, M. Owner-Petersen, "Emulation of dual-conjugate adaptive optics on an 8-m class telescope", Opt. Express, 11(18), 2231-2237, 2003.

5. Adaptive Optics for Industry and Medicine, Proceedings of the 4th International Workshop Series: Springer Proceedings in Physics, Vol. 102, Ed. Wittrock, Ulrich, 2005.

6. E.J. Fernndez, I. Iglesias, P.Artal, “Closed-loop adaptive optics in the human eye”, Opt. Lett, 26 (10), 746-748, 2001.

7. G. Vdovin, V. Kiyko, "Intracavity control of a 200-W continuous-wave NdYAG laser by a micromachined deformable mirror", Opt. Lett., 26 (11), 798-800, 2001.

8. G. Vdovin, M. Loktev, “Deformable mirror with thermal actuators”, Opt. Lett., 27 (9), 677-679, 2002.

9. J.R. Kuhn, G. Moretto, R. Racine, F. Roddier, R. Coulter, “Concepts for a Large-Aperture, High Dynamic Range Telescope", Pub. of the Astron. Soc. of the Pacific, 113, 1486-1510, 2001.

10. J.W. Hardy, Adaptive Optics for Astronomical Telescopes (Oxford University Press, Oxford, 1998).

11. S. Restaino, G. Gilbreath, D. Payne, J. Baker, T. Martinez, M. DiVittorio, D. Mozurkewich, and J. Friedman, "Results from a portable Adaptive Optics system on the 1 meter telescope at the Naval Observatory Flagsta Station", Proceedings of SPIE 4838, 1084-1090, 2003.

12. M.Le Louarn, "New challenges for adaptive optics: extremely large telescopes", Monthly notices of the Royal Astronomical society, 317 (3), 535-544, 2000.

13. E. Kibblewhite, M.F. Smutko, M. Chun, "Deformable mirrors for astronomy”, (Active and Adaptive Optical Components and Systems II, M.A. Ealey; Ed.), Proc. SPIE 1920, 115-120, 1993.

14. http://www.okotech.com

15. S.J.B Yoo, M.A. Koza, R. Bhat, C. Caneau, "1.5 $\mu \mathrm{m}$ symmetric Fabry-Perot modulators with two distinct modulation and chirp characteristics”, App. Phys. Lett., 72(25), 3246-3248, 1998. 